\title{
Observations of Comets, Minor Planets and Meteors in Japan
}

\author{
Ichiro Hasegawa \\ 2-3-11, Saidaijinogami, Nara 631, Japan
}

\section{1. $\underline{\text { Comets }}$}

In the early period of this century, some comets were discovered by Japanese amateur astronomers. Just fifty years ago, in 1937, Minoru Honda began to search for systematically comets with his $15-\mathrm{cm}$ reflector. He observed Comet Encke without an ephemeris in November, 1937, and made his firts discovery of a comet in 1940. This comet was also discovered independently by Shigeki Okabayashi (Comet Okabayashi-Honda, 1940e). Subsequently, Honda discovered Comet Friend-Reese-Honda (1941a) in 1941. Unfortunately, Okabayashi was killed at the Second World War in 1945.

After the end of the war, Honda discovered ten comets during 1947 and 1968. In 1968, he discovered three comets in a year. He has been an active observer of comets and novae. Stimulated by Honda's success, Tsutomu Seki began his comet hunting in 1950, and his long and patient efforts were rewarded with the discovery of Comet Seki, 1961f. Seki later discovered six comets, and rediscovered six short-period comets before 1981. In that year, Seizo Goto, a well-known telescope maker in Japan, donated a $60-\mathrm{cm}$ reflector. Since then, with the Goto telescope, Seki has made many astronomic observations of comets and minor planets. He has rediscovered some periodic comets and has discovered many minor planets every year.

Kaoru Ikeya discovered his first comet in 1963. In 1965, Ikeya and Seki independently discovered a sun-grazing comet. The interval between their discoveries was only 15 minutes. Comet Ikeya-Seki (1965f) had a great influence upon amateur astronomers, and observation and searching for comets became more active in Japan.

In the morning of May the 1st in 1968, six Japanese amateur observers discovered a 7magnitude comet independently. It was named Comet Tago-Honda-Yamamoto (1968a) after three of the discoverers. At the present moment, there are seven comets named after three Japanese amateurs, and four after two Japanese comet hunters. It may be noteworthy that in 1975, Hiroaki Mori discovered two comets on the same morning.

All the observers mentioned above made visual discoveries. Since 1970, however, some observers have come to use photographic techniques to discover comets. Nobuhisa Kojima (1970r 
and 1972j), Toshihiko Ikemura (1975b), Takeshi Urata and Tsuneo Niijima (1986o) discovered their comets (fainter than 12th magnitude) by photographic observations.

Since 1971, comet observers in Japan gather once a year to have a comet conference. The convention held in March this year invited Dr B.G. Marsden, a comet expert from the Smithsonian Institution. It is estimated that more than fifty Japanese comet hunters are searching for comets every night. The statistics of comet discoveries are given in the accompanying table.

Comet Discovery Statistics (1961-1986)

\begin{tabular}{|c|c|c|c|}
\hline Year & $\begin{array}{l}\text { Discovery of } \\
\text { New comets } \\
\text { (Total) }\end{array}$ & $\begin{array}{l}\text { Discovered by } \\
\text { Amateurs } \\
\text { (Breakdown) }\end{array}$ & $\begin{array}{l}\text { Discovered by } \\
\text { Japanese amateurs } \\
\text { (Breakdown) }\end{array}$ \\
\hline 1961 & 3 & 2 & 1 \\
\hline 1962 & 2 & 2 & 2 \\
\hline 1963 & 5 & 2 & 1 \\
\hline 1964 & 3 & 2 & 2 \\
\hline 1965 & 5 & 2 & 1 \\
\hline 1966 & 4 & 1 & 1 \\
\hline 1967 & 4 & 3 & 2 \\
\hline 1968 & 7 & 5 & 3 \\
\hline 1969 & 5 & 3 & 2 \\
\hline 1970 & 6 & 5 & 4 \\
\hline 1971 & 1 & 1 & 1 \\
\hline 1972 & 6 & 2 & 1 \\
\hline 1973 & 8 & 0 & 0 \\
\hline 1974 & 5 & 2 & 0 \\
\hline 1975 & 13 & 8 & 5 \\
\hline 1976 & 5 & 2 & 0 \\
\hline 1977 & 8 & 1 & 0 \\
\hline 1978 & 11 & 7 & 2 \\
\hline 1979 & 6 & 3 & 0 \\
\hline 1980 & 10 & 4 & 0 \\
\hline 1981 & 7 & 0 & 0 \\
\hline 1982 & 2 & 1 & 0 \\
\hline 1983 & 13 & 2 & 2 \\
\hline 1984 & 12 & 5 & 2 \\
\hline 1985 & 6 & 1 & 0 \\
\hline 1986 & 10 & 4 & 1 \\
\hline Total & 167 & 70 & 33 \\
\hline
\end{tabular}

\section{Minor Planets}

Recently, about seven or eight years ago, Japanese amateurs became able to use larger instruments to make photographic observations. They use telescopes larger than $30-\mathrm{cm}$, some $60-\mathrm{cm}$ or greater. They can now observe fainter minor planets easily near opposition. The most successful discoverers of minor planets are Seki, Suzuki, Urata, Niijima and Kizawa. 
Amateur orbit calculators also devote their efforts to determining the orbits of minor planets, and to identifying their perturbations, and have made fruitful results in confirming minor planets to be numbered at the Minor Planet Center of the IAU. Hideo Oishi, Syuichi Nakano, Takeshi Urata, Toshimasa Furuta and Takao Kobayashi are the most keen and successful computers. Nakano also determines comet orbits and makes predictions of short-period comets every year.

Facilities of the Tokyo Astronomical Observatory for communicating and confirmating discovery observations are most valuable and are encouraging the Japanese amateur activities.

\section{Meteors}

In 1920, the Oriental Astronomical Association was founded by Professor Issei Yamamoto (1889-1959). One of the most active fields of the OAA has been meteor observations. Kojiro Komaki (1903-1969) made his first observations of meteors in 1921, and led the observations of Leonid Meteors in 1933. His most important observation seems to be that of the great apparition of Lyrids in April, 1945. It occured at the end of the Second World War and because of the locality of meteor apparition, no other meteor observer witnessed this remarkable meteor shower.

Komaki organized the Nippon Meteor Society in 1956. This society is the most active group of the amateur astronomers in Japan. The Japanese meteor observers work with many kinds of techniques to observe meteors, such as the traditional visual observations, the telescopic and photographic ones. More than one hundred photographic meteors are observed by single or double stations, and their heliocentric orbits determined, in a year. Recently, the association of the Monocerotid meteors in December with Periodic Comet Mellish (1917 I) was confirmed by photographic observations.

Meteor echoes of FM-radio waves are observed and recorded automatically by microcomputers. The great shower of Draconids in October, 1985 was also recorder by FM-radio echoes as well as by visual observations.

\section{Novae}

At present, some Japanese amateurs are searching for galactic novae photographically, and extragalactic supernovae visually and/or photographically. In 1936, Kasuaki Gomi discovered a nova visually. He is one of the veteran observers of variable stars in Japan. Okabayashi also discovered another nova in 1936 .

About 20 years ago, Honda began to take patrol photographs to search for novae. Honda and Yoshiyuki Kuwano have discovered a lot of novae. Matsuo Sugano also discovered one comet, one peculiar variable star and one nova during the past four years.

We are sure that discoveries and observations of comets, minor planets, meteors, novae and supernovae by Japanese amateurs will continue to increase and thus contribute to astronomy. 